Supplement of Earth Syst. Sci. Data, 12, 1217-1243, 2020

https://doi.org/10.5194/essd-12-1217-2020-supplement

(C) Author(s) 2020. This work is distributed under

the Creative Commons Attribution 4.0 License.

(c) (1)

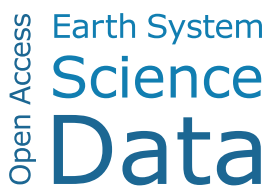

Supplement of

\title{
Annual dynamics of global land cover and its long-term changes from 1982 to 2015
}

\section{Han Liu et al.}

Correspondence to: Peng Gong (penggong@tsinghua.edu.cn) and Jie Wang (sohuwangjie@163.com)

The copyright of individual parts of the supplement might differ from the CC BY 4.0 License. 


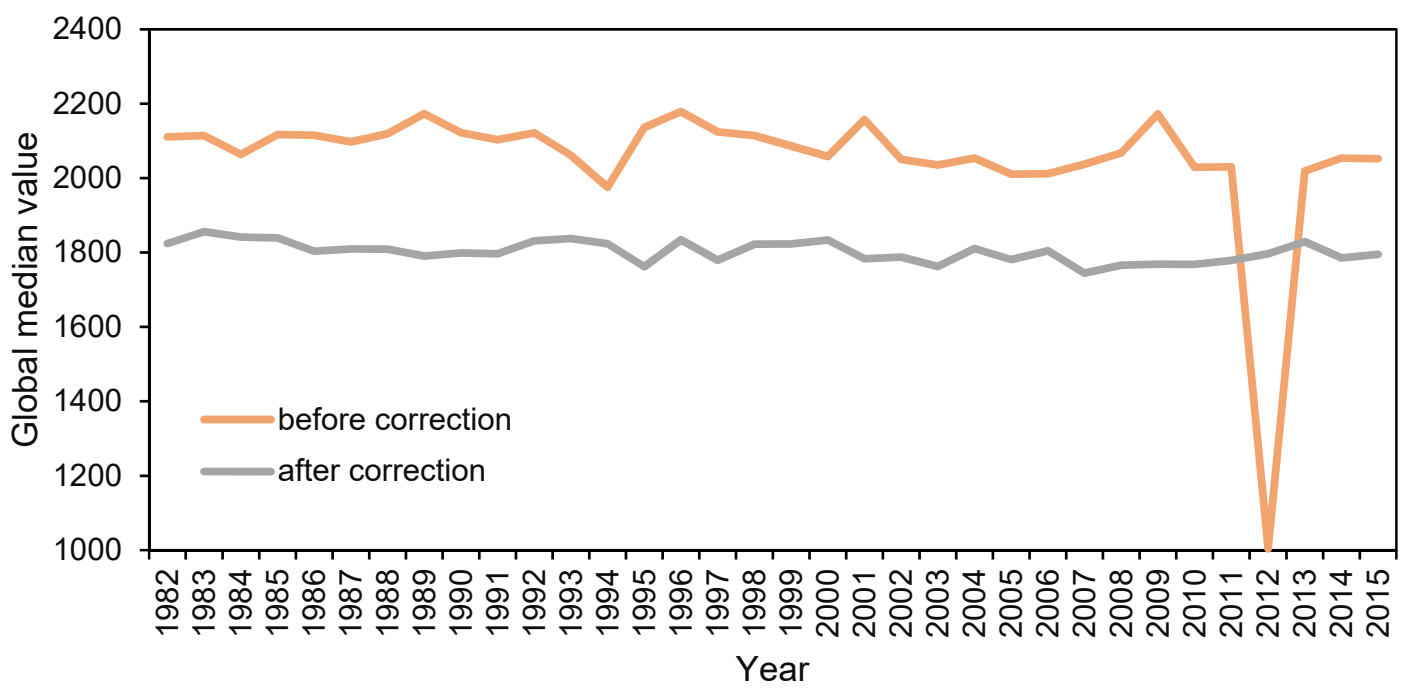

Figure S1: Global median value time series of GLASS ABD_WSA_VIS before and after the end-member correction with reference to MODIS. 


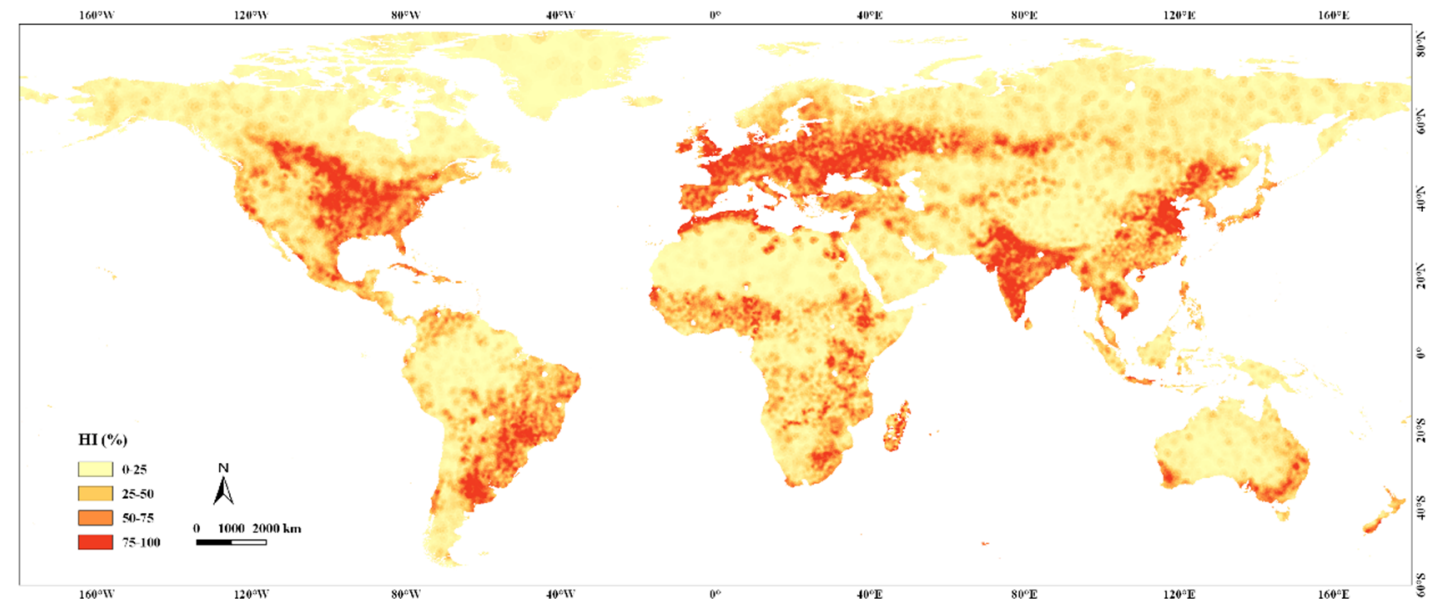

Figure S2: The geographical distribution of the spatial interpolation results of human impact where the darker color indicates a value closer to 100 and a higher human impact. 

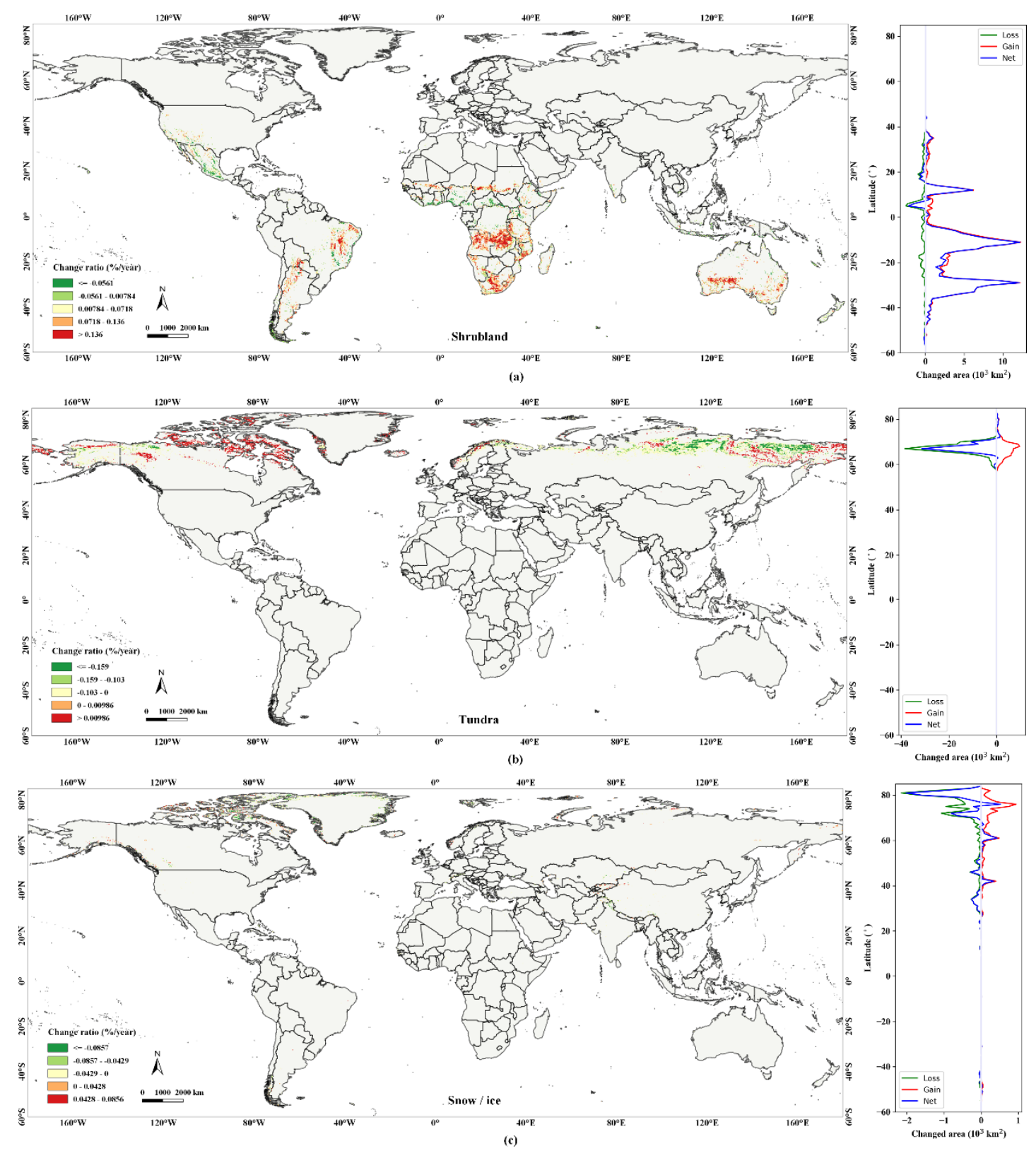

Figure S3: The geographical distribution of global regions with significant land cover change during 19822015, and the summarized results along latitudinal gradients for each class, (a) shrubland, (b) tundra and (c) snow/ice. 
Table S1: Statistical results of change analysis for shrubland (on the scale of continents). Annual change slope and its $95 \%$ confidence interval are given by Thei-sen estimator, p-value and trend information from a Mann-

Kendall test. Gain and Loss areas are summarized results relating to the whole time series.

\begin{tabular}{llllllll}
\hline Continent & $\begin{array}{l}\text { Slope }\left(10^{3}\right. \\
\mathrm{km} / \mathrm{year})\end{array}$ & $\begin{array}{l}\text { Lower }\left(10^{3}\right. \\
\mathrm{km} / \text { year })\end{array}$ & $\begin{array}{l}\text { Upper }\left(10^{3}\right. \\
\left.\mathrm{km}^{2} / \text { year }\right)\end{array}$ & $\mathrm{p}$ & Trend & $\begin{array}{l}\text { Gain }\left(10^{3}\right. \\
\left.\mathrm{km}^{2}\right)\end{array}$ & $\begin{array}{l}\text { Loss }\left(10^{3}\right. \\
\left.\mathrm{km}^{2}\right)\end{array}$ \\
\hline Africa & 47.4 & 16.1 & 74.8 & 0.0030 & increasing & 120 & -11 \\
Asia & -0.2 & -1.4 & 1.0 & 0.8125 & no trend & 1 & -1 \\
Europe & 0.0 & 0.0 & 0.0 & 0.7561 & no trend & 0 & 0 \\
North America & 0.5 & -3.0 & 5.0 & 0.8356 & no trend & 8 & -7 \\
South America & 17.8 & -0.5 & 34.7 & 0.0618 & no trend & 38 & -6 \\
Oceania & 19.9 & 3.9 & 36.2 & 0.0150 & increasing & 38 & -2 \\
Global & 75.6 & 26.1 & 125.3 & 0.0017 & increasing & 207 & -28 \\
\hline
\end{tabular}


Table S2: Statistical results of change analysis for tundra (on the scale of continents). Annual change slope and its $95 \%$ confidence interval are given by Thei-sen estimator, p-value and trend information from a Mann-

Kendall test. Gain and Loss areas are summarized results relating to the whole time series.

\begin{tabular}{llllllll}
\hline Continent & $\begin{array}{l}\text { Slope }\left(10^{3}\right. \\
\left.\mathrm{km}^{2} / \mathrm{year}\right)\end{array}$ & $\begin{array}{l}\text { Lower }\left(10^{3}\right. \\
\left.\mathrm{km}^{2} / \text { year }\right)\end{array}$ & $\begin{array}{l}\text { Upper }\left(10^{3}\right. \\
\left.\mathrm{km}^{2} / \mathrm{year}\right)\end{array}$ & $\mathrm{p}$ & Trend & $\begin{array}{l}\text { Gain }\left(10^{3}\right. \\
\left.\mathrm{km}^{2}\right)\end{array}$ & $\begin{array}{l}\text { Loss }\left(10^{3}\right. \\
\left.\mathrm{km}^{2}\right)\end{array}$ \\
\hline Africa & 0.0 & 0.0 & 0.0 & 1.0000 & no trend & 0 & 0 \\
Asia & -46.7 & -66.6 & -25.3 & 0.0002 & decreasing & 24 & -132 \\
Europe & -4.1 & -6.8 & -2.0 & 0.0015 & decreasing & 3 & -12 \\
North America & 11.4 & 0.6 & 21.5 & 0.0408 & increasing & 42 & -22 \\
South America & 0.0 & 0.0 & 0.0 & 1.0000 & no trend & 0 & 0 \\
Oceania & 0.0 & 0.0 & 0.0 & 1.0000 & no trend & 0 & 0 \\
Global & -42.0 & -63.7 & -20.9 & 0.0019 & decreasing & 71 & -167 \\
\hline
\end{tabular}


Table S3: Statistical results of change analysis for snow/ice (on the scale of continents) . Annual change slope and its $95 \%$ confidence interval are given by Thei-sen estimator, p-value and trend information from a Mann-

Kendall test. Gain and Loss areas are summarized results relating to the whole time series.

\begin{tabular}{llllllll}
\hline Continent & $\begin{array}{l}\text { Slope }\left(10^{3}\right. \\
\left.\mathrm{km}^{2} / \mathrm{year}\right)\end{array}$ & $\begin{array}{l}\text { Lower }\left(10^{3}\right. \\
\left.\mathrm{km}^{2} / \text { year }\right)\end{array}$ & $\begin{array}{l}\text { Upper }\left(10^{3}\right. \\
\left.\mathrm{km}^{2} / \text { year }\right)\end{array}$ & $\mathrm{p}$ & Trend & $\begin{array}{l}\text { Gain }\left(10^{3}\right. \\
\left.\mathrm{km}^{2}\right)\end{array}$ & $\begin{array}{l}\text { Loss }\left(10^{3}\right. \\
\left.\mathrm{km}^{2}\right)\end{array}$ \\
\hline Africa & 0.0 & 0.0 & 0.0 & 0.1342 & no trend & 0 & 0 \\
Asia & -2.4 & -4.6 & -0.4 & 0.0117 & decreasing & 2 & -2 \\
Europe & -0.8 & -1.2 & -0.2 & 0.0091 & decreasing & 1 & -1 \\
North America & -12.6 & -20.6 & -6.3 & 0.0015 & decreasing & 4 & -11 \\
South America & -0.2 & -0.3 & -0.2 & 0.0000 & decreasing & 0 & 0 \\
Oceania & 0.0 & -0.1 & 0.0 & 0.0856 & no trend & 0 & 0 \\
Global & -19.2 & -27.6 & -9.1 & 0.0003 & decreasing & 8 & -16 \\
\hline
\end{tabular}

